M. Morishita

Nagoya Math. J.

Vol. 124 (1991), 133-144

\title{
ON S-CLASS NUMBER RELATIONS OF ALGEBRAIC TORI IN GALOIS EXTENSIONS OF GLOBAL FIELDS
}

\author{
MASANORI MORISHITA
}

\section{Introduction}

As an interpretation and a generalization of Gauss' genus theory on binary quadratic forms in the language of arithmetic of algebraic tori, Ono [O2] established an equality between a kind of "Euler number $E(K ! k)$ " for a finite Galois extension $K / k$ of algebraic number fields and other arithmetical invariants associated to $K / k$. His proof depended on his Tamagawa number formula [01] and Shyr's formula [Sh] which follows from the analytic class number formula of a torus. Later, two direct proofs were given by Katayama [K] and Sasaki [Sa].

In this paper, we generalize Ono's formula to $S$-arithmetical one, including the function field case, and give a new direct proof using Nisnevich cohomology. We also give a formula by applying our method to a similar exact sequence of tori associated to two linearly disjoint Galois extensions $K_{1}, K_{2} / k$ of global fields. We think that Nisnevich cohomology is a natural and suitable tool to connect class sets of affine group schemes and etale (or flat) cohomology and to study their functorial behavior. ([N1] and [N2]).

The contents of this paper are as follows. In $\S 1$ we introduce our invariants $E_{S}(K / k)$ and $E_{S}\left(K_{1}, K_{2} / k\right)$, and in $\S 2$ we state our main results. In $\S 3$ we prove the surjectivity of the norm map. In $\S 4$ we give a brief review of Nisnevich topology and cohomology for our purpose. In $\S 5$ we prove our theorems, and in $\S 6$ we discuss some examples.

Acknowledgements. I express my sincere gratitude to Professor Yevsey A. Nisnevich who informed me of his cohomology and gave valuable advice. Especially, I owe to him an important remark on the surjectivity of the norm map in $\S 3$. I also thank Professor Takashi Ono for suggesting

Received December 10, 1990. 
me a problem, a vast generalization of Gauss' genus theory using (nonabelian) algebraic groups. I hope to come back to this beautiful problem some time in the future.

\section{§1. The invariants $E_{S}(K / k)$ and $E_{S}\left(K_{1}, K_{2} / k\right)$}

Let $k$ be a global field. For a place $v$ of $k$, let $k_{v}$ denote the completion of $k$ at $v$ and $\mathcal{O}_{v}$ the ring of integers in $k_{v}$. Let $S$ be a finite set of places of $k$ which is non-empty and contains all archimedean places, $S_{\infty}$. Let $\mathcal{O}_{S}$ be the ring of $S$-integers in $k, \mathcal{O}_{S}=\bigcap_{v \notin S} \mathcal{O}_{v}$, and put $X:=$ $\operatorname{Spec} \mathcal{O}_{S}$. For a finite set of places $P$ of $k$ containing $S$, we put

$$
k_{A}(P):=\prod_{v \in P} k_{v} \times \prod_{v \notin P} \mathcal{O}_{v}
$$

and

$$
k_{A}:=\lim _{P} k_{A}(P) \text {. }
$$

Let $G$ be an affine group scheme of finite type over $X$ with the smooth generic fibre. We define the $S$-class set $C_{S}(G)$ of $G$ by the set of double cosets:

$$
C_{S}(G)=G\left(k_{A}(S)\right) \backslash G\left(k_{A}\right) / G(k)
$$

and call its cardinality the $S$-class number of $G$ over $X$.

Let $K, K_{1}$ and $K_{2}$ be finite Galois extensions of $k$ such that $K_{1} \cap K_{2}$ $=k$, and let $S_{K}$ and $S_{i}$ be the set of places of $K$ and $K_{i}(i=1,2)$, respectively, lying above $S$. Let $\mathcal{O}_{S_{K}}$ and $\mathcal{O}_{S_{i}}$ be the normalizations of $\mathcal{O}_{S}$ in $K$ and $K_{i}$, and put $X_{K}:=\operatorname{Spec} \mathcal{O}_{S_{K}}, X_{i}:=\operatorname{Spec} \mathcal{O}_{S_{i}}(i=1,2)$. Then, let us consider the following two exact sequences of group schemes or etale sheaves on $X$ induced by the norm maps $N$ and $N_{i}$ associated to the Galois coverings $X_{K} / X$ and $X_{i} / X(i=1,2)$ respectively:

$$
\begin{gathered}
0 \longrightarrow T \longrightarrow \prod_{X_{K} / X} \mathbf{G}_{m, X_{K}} \stackrel{N}{\longrightarrow} \mathbf{G}_{m, X} \longrightarrow 0 \\
0 \longrightarrow T^{\prime} \longrightarrow \prod_{X_{1} / X} \mathbf{G}_{m, X_{1}} \times \prod_{X_{2} / X} \mathbf{G}_{m, X_{2}} \stackrel{N_{1} \cdot N_{2}}{\longrightarrow} \mathbf{G}_{m, X} \longrightarrow 0
\end{gathered}
$$

where $\mathbf{G}_{m, Y}:=\operatorname{Spec} \mathbf{Z}\left[t, t^{-1}\right] \times_{\text {spec } \mathbf{Z}} Y$ for a scheme $Y, \prod_{z / Y}$ denotes the Grothendieck functor of scaler restriction ([D-G]), $N_{1} \cdot N_{2}$ means the product of $N_{1}$ and $N_{2}$, and $T, T^{\prime}$ are defined as the kernels of $N, N_{1} \cdot N_{2}$ respectively. For the surjectivity of $N$ and $N_{1} \cdot N_{2}$, we will give a proof in $\S 3$. 
Let us denote by $h_{k, S}, h_{K, S_{K}}, h_{K_{i}, S_{i}} h_{T, S}$ and $h_{T^{\prime}, S}$ the $S$-class numbers of $\mathbf{G}_{m, X}, \prod_{x_{K} / X} \mathbf{G}_{m, X_{K}}, \prod_{x_{i} / X} \mathbf{G}_{m, X_{i}}, T$ and $T^{\prime}$ respectively. Here, $h_{k, s}, h_{K, S}$ and $h_{K_{i}, s_{i}}$ are nothing but the $S$-class numbers of $k, K$ and $K_{i}$. Now we define our invariants $E_{S}(K / k)$ and $E_{S}\left(K_{1}, K_{2} / k\right)$ by the alternative products of the $S$-class numbers taken along the sequences (1.1) and (1.2) respectively, namely,

$$
\begin{aligned}
E_{S}(K / k): & =\frac{h_{K, S}}{h_{k, S} \cdot h_{T, S}} \\
E_{S}\left(K_{1}, K_{2} / k\right): & =\frac{h_{K_{1}, S_{1}} \cdot h_{K_{2}, S_{2}}}{h_{k, S} \cdot h_{T^{\prime}, S}} .
\end{aligned}
$$

We remark that $E_{S_{\infty}}(K / k)$ coincides with Ono's $E(K / k)$ for the number field case ([O2]).

\section{§2. Statement of results}

To state our results, let us prepare more notations. For a place $v$ of $k$, we choose a place $w$ of $K$ and a place $z$ of $K_{1} K_{2}$ lying above $v$, and denote by $w_{1}$ and $w_{2}$ the restrictions of $z$ to $K_{1}$ and $K_{2}$ respectively, and put $M_{v}:=K_{1 w_{1}} \cap K_{2 w_{2}}$. Let $\mathcal{O}_{w}$ and $\mathcal{O}_{M_{v}}$ be the ring of integers in $K_{w}$ and $M_{v}$ respectively, and let $S_{r}^{\prime}$ be the set of finite places of $k$ which ramify in $K_{1} / k$ or $K_{2} / k$. For a Galois extension $E / F$ of fields, let $E^{\prime} / F$ denote the maximal abelian subextension of $E / F$. For norm maps, we put $N:=$ $N_{K / k}, N_{i}:=N_{K_{i} / k}(i=1,2), N_{v}:=N_{K_{w} / k_{v}}$, and $N_{M_{v}}:=N_{M_{v} / k_{v}}$ for simplicity. $R^{\times}$denotes the group of invertible elements in a ring $R$. Finally, [*] denotes the cardinality of a set $*$.

Theorem. Notations being as above, we have

$$
\begin{gathered}
E_{S}(K / k)=\frac{\left[\amalg_{k}(T)\right] \prod_{v \in S}\left[K_{w}^{\prime}: k_{v}\right] \prod_{v \notin S} e\left(K_{w}^{\prime} / k_{v}\right)}{\left[K^{\prime}: k\right]\left(\mathcal{O}_{S}^{\times}: N \mathcal{O}_{S_{K}}\right)} \\
E_{S}\left(K_{1}, K_{2} / k\right)=\frac{\left[\amalg_{k}\left(T^{\prime}\right)\right] \prod_{v \in S}\left[M_{v}^{\prime}: k_{v}\right] \prod_{v \notin S} e\left(M_{v}^{\prime} / k_{v}\right)}{\left(\mathcal{O}_{S}^{\times}: N_{1} \mathcal{O}_{S_{1}}^{\times} N_{2} \mathcal{O}_{S_{2}}^{\times}\right)\left(J: J \cap \Delta^{\prime} \prod_{S \cup S_{r}^{\prime}} N_{M_{v}} M_{v}^{\times}\right)}
\end{gathered}
$$

where $J:=\prod_{v \in S} k_{v}^{\times} \times \prod_{s_{r}^{\prime} \backslash S} \mathcal{O}_{v}^{\times}, \Delta^{\prime}:=\left\{x \in k^{\times} \mid x \in N_{M_{v}} M_{v}^{\times}, v \notin S \cup S_{r}^{\prime}\right\}, e\left(K_{w}^{\prime} k_{v}\right)$ and $e\left(K_{s}^{\prime} / k_{v}\right)$ denote the ramification indices of $K_{w}^{\prime} / k_{v}$ and $M_{v}^{\prime} / k_{v}$ respectively, and the Tate-Shafarevich groups over $k, \amalg_{k}(T)$ and $\amalg_{k}\left(T^{\prime}\right)$, are given by the kernels of the natural maps:

$$
\amalg_{k}(T)=\operatorname{Ker}\left(k^{\times} / N K^{\times} \longrightarrow \prod_{v} k_{v}^{\times} / N_{v} K_{w}^{\times}\right),
$$




$$
\amalg_{k}\left(T^{\prime}\right)=\operatorname{Ker}\left(k^{\times} / N_{1} K_{1}^{\times} N_{2} K_{2}^{\times} \longrightarrow \prod_{v} k_{v}^{\times} / N_{M_{v}} M_{v}^{\times}\right) .
$$

In the following, we put $I:=J / J \cap \Delta^{\prime} \prod_{s \cup s_{r}^{\prime}} N_{M_{v}} M_{v}^{\times}$for simplicity.

Corollary 1. Assume $k$ is a number field and $S=S_{\infty}$. Then we have

$$
\begin{gathered}
E_{S_{\infty}}(K / k)=\frac{\left[\amalg_{k}(T)\right] \prod_{v} e\left(K_{w}^{\prime} / k_{v}\right)}{\left[K^{\prime}: k\right]\left(\mathcal{O}_{k}^{\times}: N \mathcal{O}_{K}^{\times}\right)} \\
E_{S_{\infty}}\left(K_{1}, K_{2} / k\right)=\frac{\left[\amalg_{k}\left(T^{\prime}\right)\right] \prod_{v} e\left(M_{v}^{\prime} / k_{v}\right)}{\left(\mathcal{O}_{k}^{\times}: N_{1} \mathcal{O}_{K_{1}}^{\times} N_{2} \mathcal{O}_{K_{2}}^{\times}\right)[I]}
\end{gathered}
$$

where we put $\mathcal{O}_{v}=k_{v}$ for $v \in S_{\infty}$, and $\mathcal{O}_{k}, \mathcal{O}_{K}$ and $\mathcal{O}_{K_{i}}$ are the rings of integers in $k, K$ and $K_{i}(i=1,2)$ respectively.

Remark. (2.3) is nothing but Ono's Theorem [O2], §2.

Corollary 2. Assume that $S$ is enough large such that $h_{k, S}=h_{K, s_{K}}=$ $h_{K_{i}, S_{i}}=h_{T, S}=h_{T^{\prime}, S}=1(i=1,2)$ and $S$ contains all ramified places of $k$. Then we have the following formulas for the Tate-Shafarevich groups.

$$
\begin{aligned}
{\left[\amalg_{k}(T)\right] } & =\frac{\left[K^{\prime}: k\right]\left(\mathcal{O}_{S}^{\times}: N \mathcal{O}_{S_{K}}^{\times}\right)}{\prod_{v \in S}\left[K_{w}^{\prime}: k_{v}\right]} \\
{\left[\Psi_{k}\left(T^{\prime}\right)\right] } & =\frac{\left(\mathcal{O}_{S}^{\times}: N_{1} \mathcal{O}_{S_{1}}^{\times} N_{2} \mathcal{O}_{S_{2}}^{\times}\right)[I]}{\prod_{v \in S}\left[M_{v}^{\prime}: k_{v}\right]}
\end{aligned}
$$

where $I=J / J \cap \Delta^{\prime} \prod_{v \in S} N_{M_{v}} M_{v}^{\times}$.

CoRollary 3. If $K / k$ is cyclic, and if $K_{1} / k$ or $K_{2} / k$ is cyclic, then we have

$$
\begin{gathered}
E_{S}(K / k)=\frac{\prod_{v \in S}\left[K_{w}: k_{v}\right] \prod_{v \notin S} e\left(K_{w} / k_{v}\right)}{[K: k]\left(\mathcal{O}_{S}^{\times}: N \mathcal{O}_{S_{K}}^{\times}\right)} \\
E_{S}\left(K_{1}, K_{2} / k\right)=\frac{\prod_{v \in S}\left[M_{v}: k_{v}\right] \prod_{v \notin S} e\left(M_{v} / k_{v}\right)}{\left(\mathcal{O}_{S}^{\times}: N_{1} \mathcal{O}_{S_{1}}^{\times} N_{2} \mathcal{O}_{S_{2}}^{\times}\right)[I]}
\end{gathered}
$$

where $e\left(K_{w} / k_{v}\right)$ and $e\left(M_{v} / k_{v}\right)$ denote the ramification indices of $K_{w} / k_{v}$ and $M_{v} / k_{v}$ respectively.

\section{§3. The surjectivity of the norm map}

Let $A$ be a Dedekind domain with the field of fractions $F$. Let $E$ be a finite Galois extension of $F$ and let $B$ be the integral closure of $A$ in $E$. Put $X:=\operatorname{Spec} A$ and $Y:=\operatorname{Spec} B$.

The purpose of this section is to show the surjectivity of the norm map: 


$$
N: \prod_{Y / X} \mathbf{G}_{m, Y} \longrightarrow \mathbf{G}_{m, X}
$$

defined in the following way. For each $U$ in $X_{\text {et }}$, define

$$
\begin{aligned}
N(U): \operatorname{Hom}_{X}\left(U, \prod_{Y / X} \mathbf{G}_{m, Y}\right) & \left.=\left(B \otimes \otimes_{A} \Gamma\left(U, \mathcal{O}_{U}\right)\right)^{\times} \longrightarrow \Gamma\left(U, \mathcal{O}_{U}\right)\right)^{\times} \\
& =\operatorname{Hom}_{X}\left(U, \mathbf{G}_{m, X}\right)
\end{aligned}
$$

by $N(U)(b \otimes \gamma):=N_{B / A}(b) \gamma^{n}$, where $N_{B / A}: B \rightarrow A$ is the norm map associated to the Galois extension $E / F$ and $n=[E: F]$.

Then, we can easily see that these maps $\{N(U)\}$ commute with restriction maps and so $N$ defines a morphism of sheaves on $X_{\mathrm{et}}$.

To show the surjectivity of $N$, let us see the map $N_{\bar{x}}$ induced on the stalks at each geometric point $\bar{x}$ associated to $x \in X$ :

$$
N_{\bar{x}}:\left(\prod_{Y / X} \mathbf{G}_{m, Y}\right)_{\bar{x}}=\left(B \otimes_{A} A_{x}^{\mathrm{sh}}\right)^{\times} \longrightarrow\left(A_{x}^{\mathrm{sh}}\right)^{\times}=\left(\mathbf{G}_{m, X}\right)_{\bar{x}}
$$

where $A_{x}^{\text {sh }}$ denotes the strict henselization of $A$ at $x$.

Let $y_{i}(1 \leq i \leq g)$ be the points in $Y$ lying above $x$ with $f=\left[\kappa\left(y_{i}\right): \kappa(x)\right]$ $=$ the degree of the extension of the residue fields, and let $B_{x}^{\text {sh }}$ be the strict henselization of $B$ at $y_{i}$. Finally let $F_{x}^{\mathrm{sh}}$ and $E_{y_{i}}^{\mathrm{sh}}$ denote the fields of fractions of $A_{x}^{\text {sh }}$ and $B_{y_{i}}^{\text {sh }}$ respectively.

Then, via a canonical isomorphism

$$
B \otimes_{A} A_{x}^{\mathrm{sh}} \sim \prod_{i=1}^{g} \prod_{j=1}^{f} B_{y_{i}, j}^{\mathrm{sh}} B_{y_{i}, j}^{\mathrm{sh}}=B_{y_{i}}^{\mathrm{sh}}
$$

we can see

$$
N_{\bar{x}}=\prod_{i=1}^{g} \prod_{j=1}^{f} N_{i, j}
$$

where $N_{i, j}: B_{y_{i}, j}^{\mathrm{sh}}=B_{y_{i}}^{\mathrm{sh}} \rightarrow A_{x}^{\mathrm{sh}}$ is the norm map associated to the Galois extension $E_{y_{i}}^{\mathrm{sh}} / F_{x}^{\mathrm{sh}}$. So, it suffices to show the surjectivity of each $N_{i, j}$ which, however, follows from that the cohomological dimension of $F_{x}^{\text {sh }} \leq 1$ ([Se], II-3.1, 4.3).

\section{§4. Nisnevich topology and class sets}

The aim of this section is to introduce a certain Grothendieck topology, called Nisnevich topology, and state a theorem which connects a class set of an affine group scheme and etale cohomology. For the details of proofs in the following, we refer to [N2].

Let $X$ be any noetherian scheme. We define a Grothendieck topology 
$X_{\mathrm{Nis}}$ on $X$ by the following: As a category, $X_{\mathrm{Nis}}$ is the same as the small etale site on $X$. A family of morphisms $\left(\phi_{i}: U_{i} \rightarrow U\right)$ in $X_{\mathrm{Nis}}$ is a covering if and only if for any $x \in U$, there exists an index $i$ and $y \in U_{i}$ such that $\phi_{i}(y)=x$ and $\kappa(y) \simeq \kappa(x)$, where $\kappa(x)$ and $\kappa(y)$ are the residue fields of $U$ and $U_{i}$ at $x$ and $y$ respectively. The cohomology $H_{\mathrm{Nis}}^{*}(X, \mathscr{F})$ for any sheaf of group $\mathscr{F}$ on $X_{\mathrm{Nis}}$ are defined in the usual way (cf. [M.A]).

Let $X=\operatorname{Spec} R, R$ is a Dedekind domain and let $G$ be an affine group scheme of finite type over $X$ with the smooth generic fibre. Then we can define the class set $C(G)$ in the obvious way. (If $X$ is ours in $\S 1$, it is $C_{S}(G)$ in $\S 1$.)

The next theorem is the main property of Nisnevich cohomology for our purpose.

THEOREM ([N1], [N2]) We have a canonical bijection

$$
H_{\mathrm{Nis}}^{1}(X, G) \simeq C(G) \text {. }
$$

Furthermore we have an exact sequence of pointed sets

$$
1 \longrightarrow C(G) \longrightarrow H_{\mathrm{et}}^{1}(X, G) \longrightarrow H_{\mathrm{Nis}}^{0}\left(X, R^{1} u_{*} G\right) \longrightarrow 1
$$

and the stalk $\left(R^{1} u_{*} G\right)_{x}$ at $x \in X$ can be computed by the formula

$$
\left(R^{1} u_{*} G\right)_{x} \simeq H_{\mathrm{et}}^{1}\left(R_{x}^{h}, G\right)
$$

where $u: X_{\mathrm{et}} \rightarrow X_{\mathrm{Nis}}$ is the morphism of sites and $R_{x}^{h}$ is the henselization of $R$ at $x$. If $G$ is commutative, then all maps are homomorphisms of groups.

Remark 1. (4.2) is an immediate consequence of (a non-abelian analogue of) the Cartan-Leray spectral sequence for $u: X_{\mathrm{et}} \rightarrow X_{\mathrm{Nis}}$, (4.1) and the fact that the cohomological dimension of $X_{\mathrm{Nis}} \leq 1$.

Remark 2. By (4.3), $H_{\mathrm{Nis}}^{0}\left(X, R^{1} u_{*} G\right)$ can be computed by the following way. Let $X^{\circ}$ be the set of all closed points of $X$. For $x \in X^{\circ}$, let

$$
\begin{gathered}
\alpha_{x}: H^{1}\left(R_{x}^{h}, G\right) \longrightarrow H^{1}\left(L_{x}^{h}, G\right) \\
\beta_{x}: H^{1}(L, G) \longrightarrow H^{1}\left(L_{x}^{h}, G\right)
\end{gathered}
$$

be the natural restrictions where $L_{x}^{h}$ is the field of fractions of $R_{x}^{h}$. Then we have

$$
H_{\mathrm{Nis}}^{0}\left(X, R^{1} u_{*} G\right)=\left\{a \in H^{1}(L, G) \mid \beta_{x}(a) \in \operatorname{Im}\left(\alpha_{x}\right) \text {, for each } x \in X^{\circ}\right\} .
$$

In the above, we can replace $L_{x}^{h}$ by $L_{v(x)}$ where $L_{v(x)}$ is the completion of $L_{x}^{h}$ with respect to $x$, because Greenberg approximation theorem [G] tells 
us the injectivity $H^{1}\left(L_{x}^{h}, G\right) \stackrel{\subset}{\longrightarrow} H^{1}\left(L_{v(x)}, G\right)$.

\section{§5. Proof of theorem}

First, we shall prove the formula (2.1). (2.2) is obtained in the similar way. In the following, notations will be as in $\S 1$ and $\S 2$. All cohomology groups are etale ones except Nisnevich ones.

By (1.1), we have a long exact sequence;

$$
\begin{gathered}
\cdots \longrightarrow H^{0}\left(X, \prod_{x_{K} / X} \mathbf{G}_{m, x_{K}}\right) \stackrel{N}{\longrightarrow} H^{0}\left(X, \mathbf{G}_{m, X}\right) \longrightarrow H^{1}(X, T) \\
\longrightarrow H^{1}\left(X, \prod_{x_{K} / X} \mathbf{G}_{m, x_{K}}\right) \stackrel{N}{\longrightarrow} H^{1}\left(X, \mathbf{G}_{m, X}\right) \longrightarrow \cdots
\end{gathered}
$$

where it is easy to see the followings:

$$
\begin{aligned}
& H^{0}\left(X, \mathbf{G}_{m, X}\right)=\mathcal{O O}_{S}^{\times}, \\
& H^{0}\left(X, \prod_{X_{K} / X} \mathbf{G}_{m, X_{K}}\right)=H^{0}\left(X_{K}, \mathbf{G}_{m, X_{K}}\right)=\mathcal{O}_{S_{K}}^{\times}, \\
& H^{1}\left(X, \mathbf{G}_{m, X}\right)=\operatorname{Pic}(X)=C_{S}\left(\mathbf{G}_{m, X}\right), \\
& H^{1}\left(X, \prod_{x_{K} / X} \mathbf{G}_{m, X_{K}}\right)=H^{1}\left(X_{K}, \mathbf{G}_{m, X_{K}}\right)=\operatorname{Pic}\left(X_{K}\right)=C_{S}\left(\prod_{x_{K} / X} \mathbf{G}_{m, X_{K}}\right) .
\end{aligned}
$$

For $H^{1}(X, T)$, by (4.1) and (4.2), we have an exact sequence

$$
0 \longrightarrow C_{S}(T) \longrightarrow H^{1}(X, T) \longrightarrow H_{\mathrm{Nis}}^{0}\left(X, R^{1} u_{*} T\right) \longrightarrow 0
$$

where $u: X_{\mathrm{et}} \rightarrow X_{\mathrm{Nis}}$ is the morphism of sites.

From (1.3) and (5.1)-(5.3), we have

$$
E_{S}(K / k)=\frac{\left[H_{\mathrm{Nis}}^{0}\left(X, R^{1} u_{*} T\right]\right)}{\left(\mathcal{O}_{S}^{\times}: N \mathcal{O}_{S_{K}}^{\times}\right)\left[\operatorname{Coker}\left(\operatorname{Pic}\left(X_{K}\right) \stackrel{N}{\longrightarrow} \operatorname{Pic}(X)\right)\right]} .
$$

For simplicity, we set

$$
\begin{gathered}
C:=\operatorname{Coker}\left(\operatorname{Pic}\left(X_{K}\right) \stackrel{N}{\longrightarrow} \operatorname{Pic}(X)\right) \\
\Gamma:=H_{\mathrm{Nis}}^{0}\left(X, R^{1} u_{*} T\right) .
\end{gathered}
$$

For $C$, in terms of idele, it is easy to see that

$$
C \simeq k_{A}^{\times} / k^{\times} J_{S} N K_{A}^{\times}
$$

where $J_{S}:=\prod_{v \in S} k_{v}^{\times} \times \prod_{v \notin S} \mathcal{O}_{v}^{\times}$and $N$ means the norm map of ideles in the obvious sense. On the other hand, the Artin reciprocity tells us a canonical isomorphism

$$
k_{A}^{\times} / k^{\times} N K_{A}^{\times} \simeq \operatorname{Gal}\left(K^{\prime} / k\right) .
$$

Since the norm map of an unramified local extension is surjective, we have 


$$
\begin{aligned}
\operatorname{Ker}\left(k_{A}^{\times} / k^{\times} N K_{A}^{\times} \longrightarrow k_{A}^{\times} / k^{\times} J_{S} N K_{A}^{\times}\right) & \simeq J_{S} / J_{S} \cap k^{\times} N K_{A}^{\times} \\
& \simeq J_{r} / J_{r} \cap \Delta \prod_{v \in S \cup s_{r}} N_{v} K_{w}^{\times}
\end{aligned}
$$

where $S_{r}$ denotes the set of all finite places of $k$ which ramify in $K / k$,

$$
J_{r}:=\prod_{v \in S} k_{v}^{\times} \times \prod_{v \in S_{r} \mid S} \mathcal{O}_{v}^{\times} \quad \text { and } \quad \Delta:=\left\{x \in k^{\times} \mid x \in N_{v} K_{w}^{\times}, v \notin S \cup S_{r}\right\} .
$$

From (5.5)-(5.7), we have

$$
[C]=\frac{\left[K^{\prime} ; k\right]}{[D]}
$$

where we put $D:=J_{r} / J_{r} \cap \Delta \prod_{v \in S \cup S_{r}} N_{v} K_{w}^{\times}$.

Finally, let us analyze $\Gamma$ by the Remark 2 in $\S 4$. We identify the closed points of $X$ and the places of $k$ outside $S$. Let

$$
\begin{aligned}
& \alpha_{v}: H^{1}\left(\mathcal{O}_{v}^{h}, T\right) \longrightarrow H^{1}\left(k_{v}, T\right) \\
& \beta_{v}: H^{1}(k, T) \longrightarrow H^{1}\left(k_{v}, T\right)
\end{aligned}
$$

be the natural restrictions for $v \notin S$ where $\mathcal{O}_{v}^{h}$ is the henselization of $\mathcal{O}_{S}$ at $v$. Here, from (1.1), we have $H^{1}\left(\mathcal{O}_{v}^{h}, T\right) \simeq \mathcal{O}_{v}^{h \times} / N \mathcal{O}_{w}^{h \times}$ and $H^{1}\left(k_{v}, T\right) \simeq$ $k_{v}^{\times} / N K_{w}^{\times}$because $\operatorname{Pic}\left(\mathcal{O}_{w}^{h}\right)=0$ and Hilbert 90 respectively, where $\mathcal{O}_{w}^{h}$ is the henselization of $\mathcal{O}_{S_{K}}$ at $w$. Hence, by the density of $\mathcal{O}_{v}^{h}$ in $\mathcal{O}_{v}$ and the openness of $N \mathcal{O}_{w}^{\times}$in $\mathcal{O}_{v}^{\times}$, we have

$$
\operatorname{Im}\left(\alpha_{v}\right) \simeq \mathcal{O}_{v}^{\times} / N \mathcal{O}_{w}^{\times} .
$$

Since $\left(\mathcal{O}_{v}^{\times}: N \mathcal{O}_{w}^{\times}\right)=e\left(K_{w}^{\prime} / k_{v}\right)$ : = the ramification index of $K_{w}^{\prime} / k_{v}$ by local class field theory, from (4.4) and (5.9), we have

$$
\Gamma=\operatorname{Ker}\left(H^{1}(k, T) \stackrel{m}{\longrightarrow} \prod_{v \notin S \cup S_{r}} H^{1}\left(k_{v}, T\right) \times \prod_{v \in S_{r} \backslash S} H^{1}\left(k_{v}, T\right) /\left(\operatorname{Im} \alpha_{v}\right)\right)
$$

where the $m$ is the natural restriction.

So, we are led to look at the following commutative exact diagram.

$$
\begin{aligned}
& 0 \longrightarrow \prod_{v \in S} H^{1}\left(k_{v}, T\right) \times \prod_{v \in S_{r} \backslash S} \operatorname{Im} \alpha_{v} \\
& \prod_{v \in S} k_{v}^{\times} \mid N_{v} K_{w}^{\times} \stackrel{2 !}{\times} \prod_{v \in S_{T} \backslash S} \mathcal{O}_{v}^{\times} / N_{v} \mathcal{O}_{w}^{\times} \\
& k^{\times} / N K^{\times} \quad k^{\times} / N K^{\times} \\
& \begin{array}{c}
\begin{array}{c}
\mid l \\
H^{1}(k, T) \\
1 \downarrow
\end{array} \\
\longrightarrow \prod_{v} H^{1}\left(k_{v}, T\right) \longrightarrow \prod_{v \notin S_{r} \cup S} H^{1}\left(k_{v}, T\right) \times \prod_{v \in S_{r} \backslash S}^{\lfloor!} H^{1}\left(k_{v}, T\right) / \operatorname{Im} \alpha_{v} \longrightarrow 0
\end{array} \\
& \prod_{v} k_{v}^{\times} / \stackrel{N}{v}_{v} K_{w}^{\times} \quad \prod_{v \in S_{r} \cup S} k_{v}^{\times} / N_{v} K_{w}^{\times} \times \prod_{v \in S_{r} \backslash S}^{\ell \mid} k_{v}^{\times} / \mathcal{O}_{v}^{\times} N_{v} K_{w}^{\times}
\end{aligned}
$$


where the maps in the bottom are componentwise and each isomorphism follows from (1.1) and Hilbert 90, or (5.9). Here, $\operatorname{Ker}(m)=\Gamma$ by (5.10), $\operatorname{Ker}(l)=\Psi_{k}(T)$ by the definition, and $k_{v}^{\times} / N K_{w}^{\times} \simeq G\left(K_{w}^{\prime} / k_{v}\right)$ by local class field theory. To apply snake lemma to the above diagram, let us see

$$
\begin{aligned}
& \operatorname{Ker}(\operatorname{Coker}(l) \longrightarrow \operatorname{Coker}(m)) \\
& \quad \simeq \operatorname{Ker}\left(\prod_{v} k_{v}^{\times} / k^{\times} \prod_{v} N_{v} K_{w}^{\times} \longrightarrow \prod_{v \notin S} k_{v}^{\times} / k^{\times}\left(\prod_{v \notin S \cup S_{r}} N_{v} K_{w}^{\times} \times \prod_{v \in S_{r} \backslash S} \mathcal{O}_{v}^{\times} N_{v} K_{w}^{\times}\right)\right) \\
& \quad \simeq J_{r} / J_{r} \cap \Delta \prod_{v \in S \cup S_{r}} N_{v} K_{w}^{\times}=D((5.8))
\end{aligned}
$$

and so we have

$$
\Gamma=\frac{\left[\amalg_{k}(T)\right] \prod_{v \in S}\left[K_{w}^{\prime}: k_{v}\right] \prod_{v \notin S} e\left(K_{w}^{\prime} / k_{v}\right)}{[D]}
$$

Together with (5.4), (5.8) and (5.12), we get (2.1).

Next, let us turn to the proof of (2.2). As in the above case, by the long exact sequence associated to (1.2), we have

$$
E_{S}\left(K_{1}, K_{2} / k\right)=\frac{\left[\Gamma^{\prime}\right]}{\left(\mathcal{O}_{S}^{\times}: N_{1} \mathcal{O}_{S_{1}}^{\times} N_{2} \mathcal{O}_{S_{2}}^{\times}\right)\left[C^{\prime}\right]}
$$

where

$$
\begin{gathered}
C^{\prime}:=\operatorname{Coker}\left(\operatorname{Pic}\left(X_{1}\right) \times \operatorname{Pic}\left(X_{2}\right) \stackrel{N_{1} N_{2}}{\longrightarrow} \operatorname{Pic}(X)\right) \\
\Gamma^{\prime}:=H_{\mathrm{Nis}}^{0}\left(X, R^{1} u_{*} T^{\prime}\right) .
\end{gathered}
$$

In terms of idele, $C^{\prime} \simeq k_{A}^{\times} / k^{\times} J_{S} N_{1} K_{1 A}^{\times} N_{2} K_{2 A}^{\times}$, however, by class field theory, the subgroup $k^{\times} N_{1} K_{1 A}^{\times} N_{2} K_{2 A}^{\times}$of $k_{A}^{\times}$corresponds to the maximal abelian subextension of $K_{1} \cap K_{2}$ over $k$, which is $k$ itself by our assumption. Hence $C^{\prime}=\{1\}$.

To analyze $\Gamma^{\prime}$, note that

$$
H^{1}\left(k_{v}, T^{\prime}\right) \simeq k_{v}^{\times} / N_{v} M_{v}^{\times}
$$

which follows from (1.2). Hilbert 90 and local class field theory. The rest of argument is quit same as in the case of $\Gamma$. So we left the details to the reader. The reason that the term $I$ appears in (2.2) may be clear by (5.11).

Concerning the corollaries, corollaries 1, 2 are immediate consequences of theorem. (2.7) and (2.8) i.e., the vanishing of $\Psi_{k}(T)$ and $\Psi_{k}\left(T^{\prime}\right)$, follow from the Tchebotareff density theorem and $[\mathrm{H}]$, Proposition 3.3. 


\section{§ 6. Examples}

For some examples of $E_{S_{\infty}}(K / k)$ for the number field case, we refer to [O2], $\S 5$.

EXAMPLE 1. (Gauss' genus formula for the function field case) Suppose that $k=\mathbf{F}_{q}(T)$, the rational function field over a finite field with $q$ elements, and $K$ is a quadratic extension of $k$ and suppose that $S=\{\infty\}$, where $\infty$ denotes the place of $k$ corresponding to the pole of $T$. For simplicity, we assume that the characteristic of $k$ is different from 2 .

According to E. Artin [E.A], let us say that $K$ is real when $\infty$ is decomposed into two distinct places in $K$, and $K$ is imaginary, otherwise. By $S$-unit theorem or [E.A], $\S 14$,

$$
\mathcal{O}_{S}^{\times}=\mathbf{F}_{q}^{\times}, \quad \mathcal{O}_{S_{K}}^{\times} \simeq \begin{cases}\mathbf{F}_{q}^{\times} \times \mathbf{Z} & K \text { is real. } \\ F_{q}^{\times} & K \text { is imaginary. }\end{cases}
$$

and so

$$
\left(\mathcal{O}_{S}^{\times}: N \mathcal{O}_{S_{K}}^{\times}\right)= \begin{cases}1 & K \text { is real and } N \mathcal{O}_{S_{K}}^{\times}=\mathbf{F}_{q}^{\times} . \\ 2 & K \text { is real and } N \mathcal{O}_{S_{K}}^{\times}=\left(\mathbf{F}_{q}^{\times}\right)^{2} \text { or } K \text { is imaginary. }\end{cases}
$$

Therefore (2.7) yields

$$
E_{S}(K / k)= \begin{cases}2^{t_{K}-2} & K \text { is real and } N \mathcal{O}_{S_{K}}^{\times}=\left(\mathbf{F}_{q}^{\times}\right)^{2} . \\ 2^{t_{K}-1} & K \text { is real and } N \mathcal{O}_{S_{K}}^{\times}=\mathbf{F}_{q}^{\times} \text {or } K \text { is imaginary. }\end{cases}
$$

where $t_{K}$ is the number of places of $k \neq \infty$ which ramify in $K / k$. This is the exact analogue of the number field case; [O2], §5, Example 2. See also [E.A] $\S 11$.

ExAmple 2. (Cyclotomic function fields) We refer to [G-R] for some properties about cyclotomic function fields, which we shall use below.

Suppose that $k=\mathbf{F}_{q}(T)$ and $K=k\left(\Lambda_{f}\right)$, the $f$-th cyclotomic function field, where $f$ is an irreducible polynomial of degree $d$ in $R:=\mathbf{F}_{p}[T]$. Suppose $S=\{\infty\}$. It is known that $K / k$ is a cyclic extension whose Galois group is isomorphic to $(R /(f))^{\times}$, which is the cyclic group of order $q^{d-1}$. Let $K^{+}$be the fixed field of $\mathbf{F}_{q}^{\times}=R^{\times} \subset(R /(f))^{\times}$. For the ramifications and units, the following analogies of cyclotomic number fields are known.

(1) Every place except $(f)$ and $\infty$ is unramified in $K / k . \quad(f)$ is totally ramified in $K / k . \quad \infty$ splits totally in $K^{+} / k$ and each of these places of $K^{+}$ 
is totally ramified in $K / K^{+}$.

(2) $\mathcal{O}_{S_{K}}^{\times}=\mathcal{O O}_{S_{K+}}^{\times}$.

Since $\mathcal{O}_{S_{K+}}^{\times} \simeq \mathbf{F}_{q}^{\times} \times \mathbf{Z}^{d /(q-1)}$ by (1) and $S$-unit theorem, we have, by (2),

$$
\left(\mathcal{O}_{S_{K+}}^{\times}: N_{K / k} \mathcal{O}_{S_{K}}^{\times}\right)=\left(\mathcal{O}_{S_{K+}}^{\times}:\left(\mathcal{O}_{S_{K+}}^{\times}\right)^{q-1}\right)=(q-1)^{d /(q-1)+1}
$$

and

$$
\left(\mathcal{O}_{S}^{\times}: N_{K / k} \mathcal{O}_{S_{K}}^{\times}\right)=\left(\mathbf{F}_{q}^{\times}:\left(N_{K+/ k} \mathcal{O}_{S_{K+}}^{\times}\right)^{q-1}\right)=q-1 .
$$

Therefore, by (1) again, (2.7) yields

$$
E_{S}(K / k)=E_{S}\left(K / K^{+}\right)=1 .
$$

These are exact analogues of the cyclotomic number field case [02], §5, Examples 4, 5. Moreover the formula tells us that in the case of $K / K^{+}$, $h_{r, S}$ can be regarded as the "- part" of $h_{K, s}$.

EXAMPLE 3. Let $p_{1}$ and $p_{2}$ be distinct prime numbers and let $n_{1}$ and $n_{2}$ be integers such that $p_{1}^{n_{1}}$ and $p_{2}^{n_{2}} \geq 3$. Suppose that $k=\mathbf{Q}, K_{1}=\mathbf{Q}\left(\zeta_{p_{1}^{n_{1}}}\right)$ and $K_{2}=\mathbf{Q}\left(\zeta_{p_{2}^{n_{2}}}\right)$ where $\zeta_{p_{1}^{n_{1}}}$ and $\zeta_{p_{2}^{n_{2}}}$ denote primitive $p_{1}^{n_{1}}$-th and $p_{2}^{n_{2}}$-th roots of 1 , and suppose $S=S_{\infty}=\{\infty\}$. It is easy to see that $M_{\infty}=\mathbf{C}, M_{v}=\mathbf{Q}_{v}$ for $v \in S_{r}^{\prime}$ and $-1 \in \Delta^{\prime}$. So, $I=\{1\}$. Furthermore, $\mathcal{O}_{S}^{\times}=\{ \pm 1\}$ and $N_{1} \mathcal{O}_{S_{1}}^{\times}$ $=N_{2} \mathcal{O}_{S_{2}}^{\times}=\{1\}$. Therefore, by (2.8), we have

$$
E_{S_{\infty}}\left(K_{1}, K_{2} / k\right)=1 \text {. }
$$

In other word, $h_{K_{1}} \cdot h_{K_{2}}=h_{T^{\prime}}$ where $h_{K_{1}}, h_{K_{2}}$ and $h_{T^{\prime}}$ are the class numbers of $K_{1}, K_{2}$ and $T^{\prime}$ respectively.

\section{REFERENCES}

[E.A] E. Artin, Quadratishe Körper im Gebiete der höheren Kongruenzen. I, Math. Z., 19 (1924), 153-206.

[A-T] E. Artin and J. Tate, Class Field Theory, Harvard Univ., 1961.

[M.A] M. Artin, Grothendieck Topologies, Lecture Notes, Harvard Univ., 1962.

[D-G] M. Demazure and A. Grothendieck, Schémas en Groupes II, Lect. Notes in Math., 152, Springer, 1971.

[G-R] S. Galovich and M. Rosen, The class number of cyclotomic function fields, J. Number Theory, 13 (1981), 363-375.

[G] M. Greenberg, Rational points in henselian discrete valuation rings, Publ. Math. I.H.E.S., 31 (1966), 59-64.

[H] W. Hürlimann, On algebraic tori of norm type. Comment. Math. Helv., 59 (1984), 539-549.

[K] S. Katayama, $E(K / k)$ and other arithmetical invariants for finite Galois extensions, Nagoya Math. J., 114 (1989), 135-142.

[N1] Ye. A. Nisnevich, Arithmetic and cohomology invariants of semisimple group 
schemes and compactifications of locally symmetric spaces, Funct. Anal. Appl., 14. (1980), no. 1, 61-62.

[N2] - On certain arithmetic and cohomological invariants of semisimple groups, preprint, 1989.

[01] T. Ono, On the Tamagawa number of algebraic tori. Ann. of Math., 78 (1963), $47-73$.

[02] - On some class number relations for Galois extensions. Nagoya Math. J., 107 (1987), 121-133.

[Sa] R. Sasaki, Some remarks to Ono's theorem on a generalization of Gauss' genus theory, Nagoya Math. J., 111 (1988), 131-142.

[Se] J.-P. Serre, Cohomologie Galoisienne. Lect. Notes in Math., 5, Springer, 1965.

[Sh] J.-M. Shyr, Class number formulas of algebraic tori with applications to relative class numbers of certain relative quadratic extensions of algebraic number fields, Thesis, The Johns Hopkins Univ., 1974.

Department of Mathematics

The Johns Hopkins University

Baltimore, MD 21218

U.S.A. 